\title{
Bridging the Pressure Gap in Surface Science at the Atomic Level: $\mathrm{H} / \mathrm{Cu}(110)$
}

\author{
L. Österlund,* P. B. Rasmussen, P. Thostrup, E. Lægsgaard, I. Stensgaard, and F. Besenbacher ${ }^{\dagger}$ \\ Institute of Physics and Astronomy and CAMP, University of Aarhus, DK-8000 Aarhus C, Denmark
}

(Received 29 August 2000)

\begin{abstract}
The structural response of the $\mathrm{Cu}(110)$ surface to $\mathrm{H}_{2}$ gas pressures ranging from $10^{-13}$ to 1 bar is studied using a novel high-pressure scanning tunneling microscope (HP-STM). We find that at $\mathrm{H}_{2}$ pressures larger than 2 mbar the $\mathrm{Cu}(110)$ surface reconstructs into the $(1 \times 2)$ "missing-row" structure. From a quantitative analysis of the pressure dependence of the surface reconstruction, we conclude that $\mathrm{Cu}(110)$ responds identically to hydrogen at ultrahigh vacuum conditions and at atmospheric pressures. From the HP-STM data, we extract refined values for the adsorption and desorption rate constants.
\end{abstract}

DOI: $10.1103 /$ PhysRevLett.86.460

Surface science studies on single crystal model systems performed under idealized conditions, i.e., ultrahigh vacuum (UHV) and moderate temperatures, have contributed substantially to our present understanding of the elementary mechanisms governing gas-surface reactions and thereby the technologically important area of heterogeneous catalysis [1,2]. In a few cases it has even been demonstrated that new catalysts, operating at technologically relevant conditions, can be designed on the basis of such fundamental surface science insight $[3,4]$. It is therefore of utmost importance to clarify whether it is, in general, legitimate to extrapolate results obtained under such rarefied UHV conditions to high pressures and temperatures [5]. It is paramount to bridge this so-called pressure gap and to determine the structural response of surfaces to high pressures since the rates of reactions occurring on surfaces are strongly affected by surface morphology $[1,2,6,7]$. Recent efforts in this area include, e.g., the initial oxidation of $\mathrm{Ru}(0001)$ [8]. In the past it has been argued that surface and overlayer structures formed at low temperatures and UHV pressures can be very different from structures that might be thermodynamically stable only at high pressures and temperatures $[9,10]$. However, no direct imaging of the actual surface structure at atmosperic pressures at the genuine atomic level has been reported. Thus quantitative information about the response of metal surfaces to high pressures is so far lacking.

In this Letter, we present experimental results for the adsorption of hydrogen on $\mathrm{Cu}(110)$ and demonstrate the possibility of bridging the pressure gap in surface science at the atomic level. The $\mathrm{H} / \mathrm{Cu}$ system is a classical model system for activated dissociative chemisorption [11-13] and it is important from a technological viewpoint in both the methanol synthesis [14] and the water-gas shift reaction $[6,15]$. In situ, atom-by-atom imaging of a metal surface structure, and the accompanying adsorbate-induced reconstruction, in equilibrium with the gas phase at pressures up to 1 bar at room temperature, is for the first time demonstrated. A detailed comparison with previous UHV studies reveals that the $\mathrm{Cu}(110)$ surface responds identically to hydrogen at UHV pressures and at atmospheric pressures. From the high-pressure scanning tunneling mi-
PACS numbers: 68.37.Ef, 68.35.Bs, 82.65. $+\mathrm{r}$

croscope (HP-STM) data we derive quantitative values for the rate constants for adsorption and desorption that considerably improve on previously reported UHV values.

The experiments were performed in a UHV chamber equipped with a new, novel STM design, which is implemented in a high-pressure (HP) cell attached to the main UHV chamber [16]. To ensure chemical inertness, the HP cell and all metal parts of the HP-STM are electrochemically gold plated. A dedicated gas handling system was constructed in which the $\mathrm{H}_{2}$ was thoroughly cleaned by passing it through a catalyst bed $\left(\mathrm{Cu} / \mathrm{ZnO} / \mathrm{Al}_{2} \mathrm{O}_{3}\right)$ and a molecular sieve. The gas cleanliness is crucial due to the small sticking coefficient of $\mathrm{H}_{2}$ on $\mathrm{Cu}\left(s=3.4 \times 10^{-11}\right.$ at $T=298 \mathrm{~K}$ [13]), which means that if even minute amounts of impurities (such as $\mathrm{CO}$ or $\mathrm{O}_{2}$ ) were present in the gas, they would dominate the adsorption. In fact, experiments were performed with $99.9997 \%$ purity $\mathrm{H}_{2}$ gas used as delivered, and nanosized patches of impurities could immediately be detected to build up on the surface by atom-resolved STM. At the beginning of exposure, the impurity level was too low to be detected by conventional techniques such as Auger electron spectroscopy (AES) and low-energy electron diffraction (LEED). Although STM has limited chemical specificity, the ability of the STM to locally image surfaces with atomic resolution gives an unprecedented sensitivity with respect to judging the surface to be atomically clean or not, and the present study certainly demonstrates the necessity of high gas purity when performing high-pressure studies. The $\mathrm{Cu}(110)$ crystal was cleaned by standard procedures $(800 \mathrm{eV} \mathrm{Ar}$ ion sputtering followed by annealing to $820 \mathrm{~K}$ ), until the sample was clean and well ordered as determined by STM, AES, and LEED. All STM measurements were performed at $T=298 \mathrm{~K}$.

Figure 1 depicts a sequence of STM images of the $\mathrm{Cu}(110)$ surface obtained before $\mathrm{H}_{2}$ exposure (A), at an ambient $\mathrm{H}_{2}$ pressure of 1 bar (B), and finally after subsequent evacuation of the HP cell (C). It is apparent that upon $\mathrm{H}_{2}$ exposure at 1 bar the surface reconstructs into the well-known $(1 \times 2)$ missing-row structure with every second close-packed [110] $\mathrm{Cu}$ row expelled [17-20]. After evacuation of the HP cell, the surface reconstruction is 


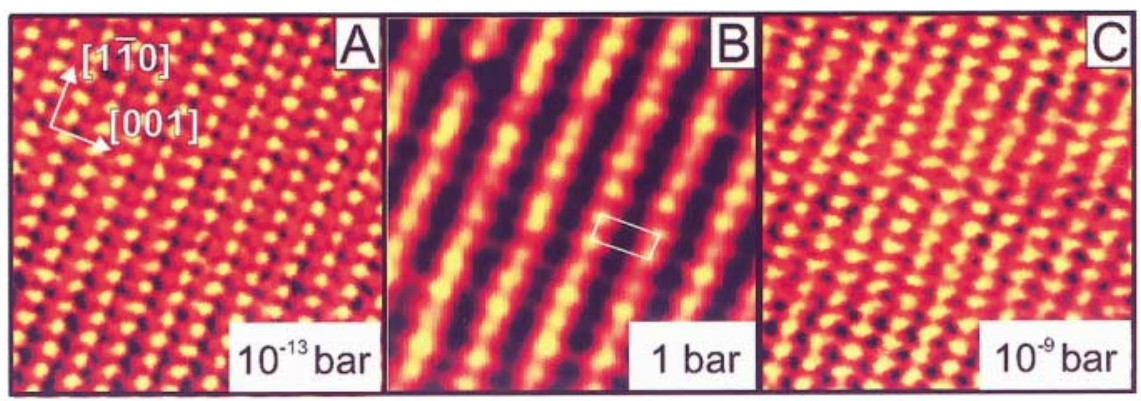

FIG. 1 (color). A sequence of atom-resolved STM images recorded at $T=298 \mathrm{~K}$ in the HP-STM depicting the Cu(110) surface (A) before $\mathrm{H}_{2}$ exposure $(I=1.42 \mathrm{nA} ; V=105 \mathrm{mV})$, (B) during exposure to 1 bar of $\mathrm{H}_{2}$ (the image is recorded $1 \mathrm{~h} 50 \mathrm{~min}$ after exposure $(I=1.55 \mathrm{nA} ; V=29.3 \mathrm{mV})$, and $(\mathrm{C})$ after a time lapse of $\sim 1500$ s directly after evacuating the HP cell $(I=1.06 \mathrm{nA}$; $V=-4.9 \mathrm{mV})$. The unit cell of the $(1 \times 2)$ missing-row structure is indicated in $(\mathrm{B})\left(2.55 \times 7.22 \AA^{2}\right)$. All figures are drawn on the same scale $\left(46 \times 45 \AA^{2}\right)$.

lifted and only a $(1 \times 1)$ surface structure is observed. This implies that during the time between evacuation of the HP cell and resumption of imaging $(\sim 1500 \mathrm{~s})$, sufficient hydrogen must have been depleted from the surface for the reconstruction to lift. Subsequent AES and STM showed that no impurities were present on the surface after the high-pressure treatment.

In order to investigate the pressure dependence of the $\mathrm{H}$-induced reconstruction, STM imaging was performed at a range of different $\mathrm{H}_{2}$ pressures between UHV and 1 bar (marked with arrows in Fig. 2). Figures 2A and 2B show two STM images obtained at 2 and $20 \mathrm{mbar} \mathrm{H}_{2}$, respectively [21]. At 2 mbar only a $(1 \times 1)$ structure is observed, while at 20 mbar the surface consists of large areas $\left(>100 \times 100 \AA^{2}\right)$ displaying the $(1 \times 2)$ reconstruction, as well as $(1 \times 1)$ domains (Fig. $2 \mathrm{~B}$ inset). This means that $(1 \times 2)$ nuclei formation starts somewhere between 2 and 20 mbar (light grey area in Fig. 2C). The sole observation of the bare $\mathrm{Cu}$ lattice at 2 mbar (Fig. 2A) - even after hours of exposure - provides a direct proof that we have well-defined conditions with no impurities present in the gas, since practically all molecules have higher sticking probability on $\mathrm{Cu}$ than $\mathrm{H}_{2}$. At 20 mbar atom-resolved imaging could be obtained only on the nonrestructured surface, i.e., on $(1 \times 1)$ domains (in Fig. $2 B$ inset). In regions where the $(1 \times 2)$ phase had nucleated, protruding domains, which were not stable over time, as well as deeper layers (dark regions), were also observed. The former protrusions ("disordered" structure in Fig. 2B) are probably due to rapidly diffusing $\mathrm{Cu}$ atoms [22] that are present on the surface during the formation of the $(1 \times 2)$ phase and which interfere with the measurements. In these experiments Ar was additionally admitted to the HP cell so that for all $\mathrm{H}_{2}$ partial pressures the total pressure was always 1 bar. This is necessary to avoid discharging between the piezoelectric scanner tube electrodes of the STM in the $10^{-3}$ to 10 mbar regime.

The binding state of the adsorbed $\mathrm{H}$ was determined by postexposure temperature-programmed desorption (TPD). Figure 3B shows a TPD spectrum after 15 min exposure at
$1 \mathrm{bar}$, recorded after a delay time between exposure and the TPD experiment of $1200 \mathrm{~s}$. The TPD spectrum is characterized by a small peak at $T=340 \mathrm{~K}$. This is interpreted as $\mathrm{H}_{2}$ originating from $\mathrm{H}$ adsorbed on the $\mathrm{Cu}(110)$ surface, consistent with previous studies $[12,23]$. The amount of $\mathrm{H}_{2}$ was calibrated by comparing with TPD spectra obtained from a saturated $\mathrm{Cu}(110)-(1 \times 2)-\mathrm{H}$ structure prepared in $\mathrm{UHV}$ by exposing the surface to atomic $\mathrm{H}$ formed by predissociation on a hot tungsten filament. The surface prepared in this manner is characterized by a sharp $(1 \times 2)$ LEED pattern and a TPD peak at $T=345 \mathrm{~K}$ and is known to saturate at a coverage of $1 \mathrm{H}$ atom per $(1 \times 2)$ surface unit cell, i.e., at a saturation coverage of $\theta_{\text {sat }}=0.5$ [19] (see Fig. 3A). Using this latter TPD peak as a reference, the amount of surface $\mathrm{H}$ in Fig. 3B is determined to be $\theta=0.10 \pm 0.02$. The low surface coverage of $\mathrm{H}$ in these TPD experiments can easily be accounted for by desorption occurring during evacuation and transfer to UHV prior to TPD when the sample is held at room temperature. By varying the time between evacuation and TPD the $\mathrm{H}$ desorption rate constant is determined to be $k_{d}=(1.2 \pm 0.1) \times 10^{-3} \mathrm{~s}^{-1}$.

A large database of UHV studies of the $\mathrm{H} / \mathrm{Cu}(110)$ system exists [12,13,17-20,23], which allows us to scrutinize the validity of the "surface science approach" to predict corresponding high-pressure phenomena. Briefly, these studies have shown that chemisorption of $\mathrm{H}$ on the $\mathrm{Cu}(110)$ surface induces the $(1 \times 2)$ missing-row reconstruction at $T>140 \mathrm{~K}[17,18]$. Experiments have shown that small $(1 \times 2)$ nuclei form already at low hydrogen coverages $\left(\theta_{\mathrm{H}} \gtrsim 0.05\right)$ [18-20]. The transformation from the reconstructed $(1 \times 2)$ to the nonreconstructed $(1 \times 1)$ surface at $T>250 \mathrm{~K}$ has also been studied and the rate constant for desorption determined $[18,19]$. The desorption is found to be of first order suggesting that the ratedetermining step is associated with restructuring of the surface rather than associative $\mathrm{H}_{2}$ desorption. The high activation barrier for $\mathrm{H}$ adsorption limits the $\mathrm{H}$ uptake onto the $\mathrm{Cu}$ surface. Therefore conventional UHV studies of molecular hydrogen adsorption have primarily been 


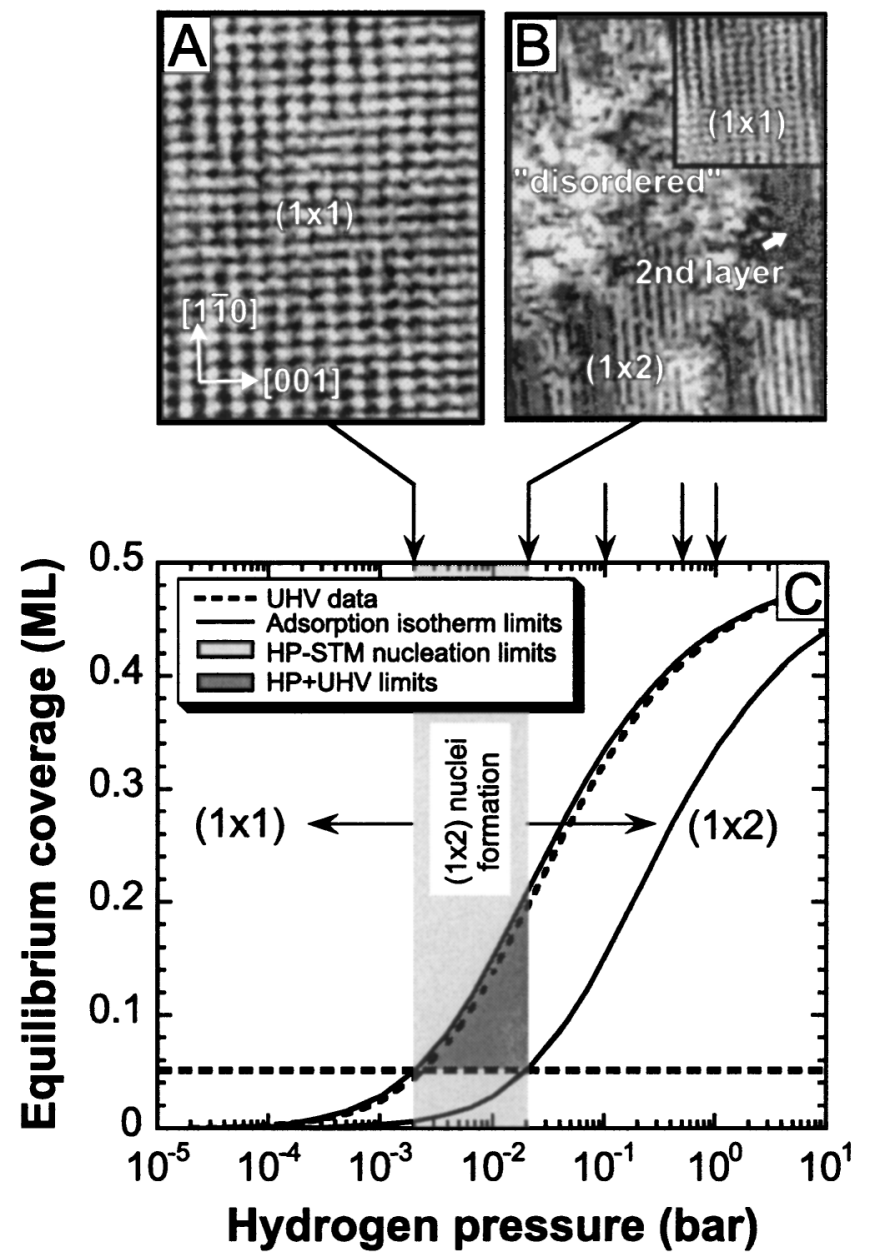

FIG. 2. (A) STM image depicting the $(1 \times 1)$ surface structure at $P_{\mathrm{H}_{2}}=2$ mbar $\left(57 \times 47 \AA^{2}\right)$, and $(\mathrm{B})$ the mixed $(1 \times 2)$ and $(1 \times 1)$ surface structures at $P_{\mathrm{H}_{2}}=20$ mbar $\left(221 \times 178 \AA^{2}\right)$. The latter structure is shown with atomic resolution in the inset in (B) $\left(40 \times 40 \AA^{2}\right)$. Patches of "disordered," protruding regions (bright color) and holes in the lattice (dark color), respectively, are visible on the surface where the $(1 \times 2)$ restructuring occurs. (C) Pressure dependence of the $(1 \times 1) \rightarrow(1 \times 2)$ $\mathrm{H}$-induced reconstruction at $T=298 \mathrm{~K}$. The arrows at the top abscissa show the different pressures at which HP-STM imaging was performed. The light grey area shows the pressure regime in which the $(1 \times 2)$ reconstruction occurs. The region enclosed by the thin solid lines marks the lower and upper limits of the equilibrium coverage vs pressure as deduced from the HP-STM measurements. The equilibrium coverage is given by $\theta_{\mathrm{eq}}=\frac{1}{2}+\alpha-\sqrt{\alpha(\alpha+1)}$, where $\alpha \equiv k_{d} / 4 k_{a} P_{\mathrm{H}_{2}}$. The horizontal dashed line indicates the low coverage limit $(\theta \gtrsim 0.05)$ for $(1 \times 2)$ nucleation, as determined from UHV studies. The curved dashed line shows the adsorption isotherm obtinaed using available UHV data (see text). Finally, the dark grey region indicates the combined high pressure and UHV limits where the surface reconstruction occurs.

done by means of molecular beam techniques, where the energy of the impinging molecules can be raised to overcome the activation barrier for adsorption [11,12,24]. However, in these latter cases no systematic study of the surface structure has been reported, although TPD

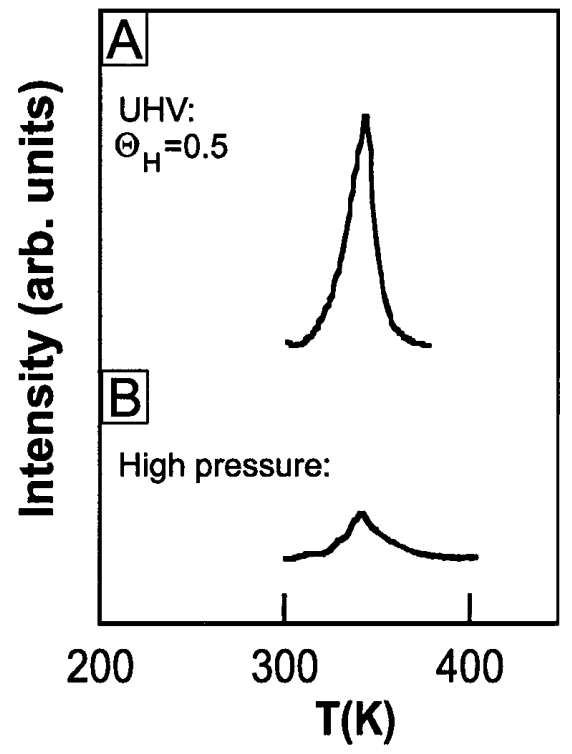

FIG. 3. TPD spectra showing the $\mathrm{H}_{2}$ desorption, $m / Z=2$. (A) Spectrum from a saturated $\mathrm{Cu}(110)\left(\theta_{\mathrm{H}}=0.5\right)$ surface prepared by adsorption of atomic $\mathrm{H}$ in UHV at $T=220 \mathrm{~K}$ and (B) from the $\mathrm{Cu}(110)$ surface after 15 min exposure to 1 bar of $\mathrm{H}_{2}$ at $T=298 \mathrm{~K}$. The latter spectrum is recorded after a time lapse of $1200 \mathrm{~s}$ between evacuation of the HP cell and transfer to UHV. The heating rate was $2 \mathrm{~K} / \mathrm{s}$.

measurements indicate that the $\mathrm{H}$ binding state is similar to the corresponding atomic $\mathrm{H}$ case [12]. In one case, high-pressure exposure has been used to compensate for the low sticking probability. In this latter study, the adsorption of a Boltzmann distributed $\mathrm{H}_{2}$ gas was studied in the range $T=470-720 \mathrm{~K}$ by reactive "titration" experiments with preadsorbed oxygen, from which the $\mathrm{H}_{2}$ sticking probability and the activation barrier for adsorption were deduced [13].

We may in a very direct way compare the rate constants for adsorption, $k_{a}$, and desorption, $k_{d}$, derived from previous UHV studies quoted above, with the high-pressure STM data. Neglecting the rate of diffusion into the bulk, which is small [25], the kinetic equation describing the adsorption and desorption is given by

$$
\frac{d \theta}{d t}=2 K_{a} P_{\mathrm{H}_{2}}\left(1-\frac{\theta}{\theta_{\mathrm{sat}}}\right)^{2}-k_{d} \theta
$$

Here $K_{a}$ is the normalized rate constant for adsorption $\left(K_{a}=k_{a} / 4\right.$ if $\left.\theta_{\text {sat }}=0.5\right)$. As discussed above, the HP-STM data provide a narrow window $\left(2 \leq P_{\mathrm{H}_{2}}<\right.$ 20 mbar) for the onset of the $(1 \times 2)$ reconstruction as a function of $\mathrm{H}_{2}$ pressure (light grey region in Fig. 2). UHV data indicate that $(1 \times 2)$ nuclei formation starts at $\theta \gtrsim 0.05$ [18-20] (horizontal dashed line in Fig. 2C). Furthermore, using Eq. (1), this implies that the H equilibrium coverage must be $\theta_{\mathrm{eq}} \lesssim 0.05$ at $P_{\mathrm{H}_{2}}=2 \mathrm{mbar}$, and $0.05 \lesssim \theta_{\mathrm{eq}}<0.21$ at $P_{\mathrm{H}_{2}}=20 \mathrm{mbar}$, or equivalently $4.1 \times 10^{-3} / P_{\mathrm{H}_{2}}[\mathrm{bar}]<\alpha<4.1 \times 10^{-2} / P_{\mathrm{H}_{2}}[$ bar $]$, where $\alpha \equiv k_{d} / 4 k_{a} P_{\mathrm{H}_{2}}$. Inserting these limits, we obtain the 
region enclosed by the thin solid lines in Fig. 2C. This reduces the region in pressure-coverage space where the $(1 \times 2)$ reconstruction occurs to the dark grey region in Fig. 2C. The HP-STM data reported here considerably narrow down the uncertainty in $\alpha$ (or $k_{d} / k_{a}$ ). Using the value for $k_{d}$ determined from the post-TPD measurements we find that $7.3 \times 10^{-3}<k_{a}<7.3 \times 10^{-2} \mathrm{~s}^{-1} \mathrm{bar}^{-1}$, which is in good agreement with previously reported values for $k_{a}=3.4 \times 10^{-2} \mathrm{~s}^{-1} \mathrm{bar}^{-1}$ [13]. Scrutinizing the rate constants for desorption reported from UHV studies, which show significant spread $[12,18,19]$, we find the best agreement with $k_{d}=6.6 \times 10^{-4} \mathrm{~s}^{-1}$ [19], which is consistent with the $\alpha$ limits imposed by the HP-STM data and the reported $k_{a}$ values. In Fig. $2 \mathrm{C}$ is shown the adsorption isotherm using this latter value of $k_{d}$ (curved dashed line). It is clear that the UHV data show remarkably good agreement with the high-pressure data reported here.

In conclusion, we have bridged the pressure gap at the ultimate resolution, i.e., on an atom-by-atom basis. By the use of a novel high-pressure STM, we have shown that for the chosen system, $\mathrm{H} / \mathrm{Cu}(110)$, the surface reconstructs into the $(1 \times 2)$ missing-row structure at $\mathrm{H}_{2}$ partial pressures $>2$ mbar and thus responds identically to a reactive gas at UHV and atmospheric pressures. The results imply that raising the pressure is equivalent to lowering the temperature, which is usually what is done in UHV studies in order to study high surface coverage structures. The HP results are in excellent agreement with available UHV data. The latter is important since it constitutes the basis for numerous well-characterized and well-controlled UHV studies aimed at elucidating related gas-surface reactions, even at "real" reaction conditions, and it provides support for the surface science approach.

We acknowledge financial support from the Danish National Research Foundation through the Center for Atomicscale Materials Physics (CAMP) and from the VELUX and Knud Højgaard Foundations.
*Present address: Competence Centre for Catalysis and Department of Applied Physics, Chalmers University of Technology, SE-412 96, Göteborg, Sweden.

${ }^{\dagger}$ Corresponding author.

Email address: fbe@ifa.au.dk

[1] G. Ertl, Surf. Sci. 299/300, 742 (1994).

[2] G. Somorjai, Surface Chemistry and Catalysis (Wiley, New York, 1994).

[3] F. Besenbacher et al., Science 279, 1913 (1998).

[4] D. A. King, Stud. Surf. Sci. Catal. 109, 70 (1997).

[5] T. Dellwig et al., Phys. Rev. Lett. 85, 776 (2000).

[6] C. T. Campbell and K.H. Ernst, in ACS Symposium Series (American Chemical Society, Washington, DC, 1992), Vol. 582, p. 130.

[7] S. Dahl et al., Phys. Rev. Lett. 83, 1814 (1999).

[8] H. Over et al., Science 287, 1474 (2000).

[9] J. A. Jensen et al., Phys. Rev. Lett. 80, 1228 (1998).

[10] P. Cernota et al., Surf. Sci. 445, 249 (2000).

[11] C. T. Rettner et al., J. Phys. Chem. 100, 13021 (1996).

[12] G. Anger, A. Winkler, and K. D. Rendulic, Surf. Sci. 220, 1 (1989).

[13] J. M. Campbell and C. T. Campbell, Surf. Sci. 259, 1 (1991).

[14] P. B. Rasmussen et al., Catal. Lett. 26, 373 (1994).

[15] C. V. Ovesen et al., J. Catal. 158, 170 (1996).

[16] E. Lægsgaard et al. (to be published).

[17] F. Besenbacher and J. K. Nørskov, Prog. Surf. Sci. 44, 5 (1993).

[18] J. Goerge et al., Surf. Sci. 289, 201 (1993).

[19] R. Spitzl et al., Surf. Sci. 239, 243 (1990).

[20] B. E. Hayden, D. Lackey, and J. Schott, Surf. Sci. 239, 119 (1990).

[21] The time to reach equilibrium coverage at low $\mathrm{H}_{2}$ pressures is quite long which is important to keep in mind when preparing the surface. At 2 mbar it is calculated to be $\sim 1800 \mathrm{~s}$.

[22] F. Besenbacher et al., Top. Catal. 1, 325 (1994).

[23] U. Bischler et al., Phys. Rev. Lett. 70, 3603 (1993).

[24] S. T. Ceyer, Science 249, 133 (1990).

[25] W. R. Wampler, T. Schober, and B. Lengeler, Philos. Mag. 34, 129 (1976). 